УДК 512.562

\title{
Strongly Algebraically Closed Lattices in $\ell$-groups and Semilattices
}

\author{
Ali Molkhasi* \\ Faculty of Mathematical Sciences \\ Farhangian University of Tehran, 51666-16471
}

Iran

Received 03.08.2017, received in revised form 05.11.2017, accepted 16.01.2018

In this article, the properties of being $\aleph_{0}$-classes of a full $\ell$-group, the set of polars of an $\ell$-group, the complemented $\ell$-ideals of a complete $\ell$-group, the set of invariant elements of a dimension ortholattice, and pseudocomplemented semilattices are studied from the perspective of model theory and their relations to strongly algebraically closed lattices are obtained.

Keywords: strongly algebraically closed lattices, $\ell$-groups, pseudocomplemented semilattices.

DOI: 10.17516/1997-1397-2018-11-2-258-263.

\section{Introduction}

Systematic study of universal algebraic geometry is done in a series of articles by V. Remeslennikov, A. Myasnikov and E. Daniyarova in ( [2-5]). In [12] J. Schmid studied algebraically closed and existentially closed distributive lattices. He proved that any Boolean lattice is algebraically closed. J. Schmid asked about the situation in which a distributive lattice is strongly algebraically closed. In [10] we defined the notion of strongly algebraically closed lattices and proved that if such a lattice is also complete Boolean and $q^{\prime}$-compact, then it is strongly algebraically closed. The current paper continues the study of [10]. We suggest [7-9], [11], and [13] in order to give a precise definition of the notion algebraically closedness of model theory and Boolean algebras.

In Section 1, it is proved that if the lattice of the $\aleph_{0}$-classes $C l(G)$ of a full $\ell$-group $G$ is complete and $q^{\prime}$-compact, then $C l(G)$ is a strongly algebraically closed lattice. Also, we prove that, if the set of polars $\mathfrak{P}(G)$ of an $\ell$-group $G$ is $q^{\prime}$-compact, then $\mathfrak{P}(G)$ is a strongly algebraically closed lattice. At the end of this section, we will show that, if the complemented $\ell$-ideals of a complete $\ell$-group is $q^{\prime}$-compact, then it is a strongly algebraically closed lattice. In the final section of this paper we study some interesting applications of strongly algebraically closed lattices about pseudocomplemented semilattices and the set of invariant elements of a dimension ortholattice.

\section{The lattice of $\aleph_{0}$-classes}

Suppose $\mathcal{L}$ is an arbitrary algebraic language and $A$ is a fixed algebra of type $\mathcal{L}$. Let $S$ be a system of equations in $A$. The set of all logical consequences of $S$ over $A$ is the radical,

*molkhasi@cfu.ac.ir

(c) Siberian Federal University. All rights reserved 
noted $\operatorname{Rad}_{A}(S)$, and $V_{A}(S)$ is the set of solutions of $S$ in $A$. For example, if $L$ is a lattice, then $\operatorname{Rad}_{L}(S)$ is the set of all lattice equations $f \approx g$ such that $V_{L}(S) \subseteq V_{L}(f \approx g)$. It is said that two lattices $L$ and $K$ are geometrically equivalent, if for any system $S$, we have $\operatorname{Rad}_{L}(S)=\operatorname{Rad}_{K}(S)$. A lattice $L$ is $q^{\prime}$-compact, if it is geometrically equivalent to any of its elementary extensions ( [4] and [3]). Let $A, B$ be two first-order structures of type $\mathcal{L}$. $A$ is called an elementary substructure of $B$ if $A \leqslant B$ and for any sentence $\Phi$ of type $\mathcal{L}_{A}$, which $\mathcal{L}_{A}$ is obtained from $\mathcal{L}$ by adding new constant symbols $a \in A$,

$$
A \models \Phi \text { iff } B \models \Phi .
$$

By a Boolean lattice we mean a complemented distributive lattice. By a Boolean algebra we mean a Boolean lattice together with the unary operation of complementation.

A lattice $L$ is called algebraically closed, if any finite consistent system of equations with coefficients from $L$, has a solution in $L$. A system $S$ with coefficients in $L$ is called consistent, if there is an extension $K$ of $L$, such that $S$ has a solution in $K$. One can generalize this definition to an arbitrary class of lattices. A lattice $L$ in a class $\mathfrak{X}$ is said to be strongly algebraically closed if every system (not necessarily finite) of equations with parameters in $L$ which has a solution in some extension $K \in \mathfrak{X}$, has already a solution in $L$ (see [10] and [12], for more details).

A group $G$ is called partially ordered group if $G$ is a additively group and the same time a partially ordered set such that for all $x, y, z \in G$

$$
x \leqslant y \Longrightarrow x+z \leqslant y+z \text { and } z+x \leqslant z+y .
$$

In addition if $G$ is a lattice, then $G$ is called a lattice ordered group (we write shortly $\ell$-group). Let us denote the positive cone of a partially ordered group $G$ by $P$ or

$$
P=\{g \in G \mid 0 \leqslant g\}
$$

and each of its members is called positive. Let $a, b$ be elements of an $\ell$-group $G$. Then

$$
a \leqslant \mathbb{N} b \Longleftrightarrow \exists n \in \mathbb{N} \text { such that } a \leqslant n b .
$$

Also,

$$
a \leqslant \aleph_{0} b \Longleftrightarrow a=\vee_{n=1}^{\infty}(n b \wedge a) .
$$

From [14], we know already that the set $\pi_{\aleph_{0}}=\left\{(a, b) \in \varrho^{2} \mid a \leqslant \aleph_{0} b\right\}$ is a preorder on the set of positive elements of the $\ell$-group $G$. For any element $a$ of $P$ we have that $k a \leqslant \aleph_{0} a$ for all $k \in \mathbb{N}$ and then $a \leqslant \aleph_{0} a$, for all $a \in P$. We will have $\pi_{\aleph_{0}}$ is reflexive. On the other hand, it is clear that in the $\ell$-group $G$ if $a \leqslant \aleph_{0} b$, then $n a \leqslant \aleph_{0} b$ for $a, b \in P$ and for all $n \in \mathbb{N}$. Therefore, we conclude that if $a \leqslant \aleph_{0} b$ and $b \leqslant \aleph_{0} c$, then $a \leqslant \aleph_{0} c$ for all $a, b, c \in P$. Now, we see the relation $\pi_{\aleph_{0}}$ is reflexive and transitive. By $\pi_{\aleph_{0}}$-classes of the $\ell$-group $G$ we mean the classes of $\pi_{\aleph_{0}}$ and will be denoted by $a^{0}$, the class of the element $a \in P$. An $\ell$-group $G$ is called a full $\ell$-group if $\bigvee_{n=1}^{\infty}(n a \wedge b)$ exists for all $a, b \in P$. A partially order group $G$ is called Archimedean if $x, y \in G$ and $n x<y$ for all integers $n$ implies $x=0$. Now, we will prove the consequences regarding the $\ell$-group $G$ if the lattice of its $\aleph_{0}$-classes is a strongly algebraically closed lattice which the lattice of $\aleph_{0}$-classes of $G$ is $q^{\prime}$-compact. Let us denote the lattice of the $\aleph_{0}$-classes of $G$ by $C l(G)$.

Theorem 1.1. Suppose that $G$ is a full $\ell$-group. If $C l(G)$ is complete and $q^{\prime}$-compact, then $\mathrm{Cl}(G)$ is a strongly algebraically closed lattice. 
Proof. We know lattice homomorphic image of the lattice of Archimedean classes is distributive. Thus, the lattice of $\aleph_{0}$-classes is distributive lattice with minimal element $0^{0}$. First we prove that an $\aleph_{0}$-class of an element is closed. Assume that for a set element $\left\{a_{\alpha}\right\}$ there exists $\vee_{\alpha} a_{\alpha}$ such that $\left(a_{\alpha}\right)^{0}=a^{0}$ for all $\alpha$. Since $a \leqslant \aleph_{0}\left(\vee_{\alpha} a_{\alpha}\right), \vee_{n=1}^{\infty}\left(n a \wedge a_{\alpha}\right)=a_{\alpha}$ and $\vee_{\alpha} a_{\alpha}=\vee_{n=1}^{\infty}\left\{n a \wedge\left(\vee_{\alpha} a_{\alpha}\right)\right\} \leqslant$ $\aleph_{0} a$. Thus, $\vee_{\alpha} a_{\alpha} \in a^{0}$ and the $\aleph_{0}$-classes are closed. Now, we show that $\operatorname{Cl}(G)$ is a relatively complemented. Certainly, we have that a relatively complemented lattice with minimal element is sectionally complemented. Therefore, we will show that $C l(G)$ sectionally complemented. Let $a^{0}$ and $b^{0}$ be arbitrary elements of $\aleph_{0}$-classes of $G$ with $a^{0} \leqslant b^{0}$. We prove $(b-c)^{0}$ is complement of $a^{0}$ in interval $\left[0^{0}, b^{0}\right]$. Since, $\aleph_{0}$-classes are closed and $n a \wedge b \in a^{0}$ for all $n \in \mathbb{N}$. So, $c^{0}=a^{0}$ or $c \in a^{0}$. We can say

$$
(b-c)^{0} \vee a^{0}=(b-c)^{0} \vee c^{0}=\{(b-c)+c\}^{0}=b^{0},
$$

and we will have

$$
b \wedge(a+c)=\vee_{n=1}^{\infty}\{b \wedge n a \wedge(a+b)\}=\vee_{n=1}^{\infty}(n a \wedge b)=c .
$$

Therefore, $(b-c) \wedge a=0$ and $(b-c)^{0} \wedge a^{0}=0^{0}$. So far we proved $C l(G)$ is a Boolean algebra. But $C l(G)$ is complete and $q^{\prime}$-compact and thus $C l(G)$ is a strongly algebraically closed lattice by ( [10], Theorem 3.5).

Let $x$ be an element of the $\ell$-group $G$. Recall that the positive part, negative part, and absolute value of $\mathrm{x}$ are defined by:

$$
x^{+}=x \vee 0, x^{-}=(-x) \vee 0,
$$

and

$$
|x|=x \vee(-x),
$$

respectively. Two elements $x, y \in X \subseteq G$ are called disjoint if $|x| \wedge|y|=e$. The disjointness of $x$ and $y$ is denoted by $x \perp y$. The subset $X$ of $G$ is called disjoint if any two distinct elements in $X$ are disjoint. The polar of $X$ is defined by

$$
X^{\perp}=\{a \in G|| a|\wedge| x \mid=e, \forall x \in X\} .
$$

Theorem 1.2. The set of polars of an $\ell$-group is a strongly algebraically closed lattice if it is $q^{\prime}$-compact.

Proof. To verify that for polars $A$ and $B, A \subseteq B$ if and only if $A \cap B^{\prime}=0$. Because for all $D, E$ the set of all convex $\ell$-subgroups of an $\ell$-group $G$ we have $D \cap E=0$ iff $D \subseteq A^{\prime}$.

On the other hand, we know that the Boolean algebra of polars of an $\ell$-group is complete, and this property is reflected in its dual space and is an essential ingredient for the representation of an archimedean $\ell$-group. So, if $G$ is an $\ell$-group, then the set of polars of a $G$ is complete Boolean algebra, Now, by ( [10], Theorem 3.5), completes the proof.

A complete $\ell$-group is $\ell$-group in which every bounded set has a least upper bound and greatest lower bound. For example, the additive group $(\mathbb{R},+)$ for all real numbers is such complete $\ell$ group.

An $\ell$-ideal $J$ of a complete $\ell$-group $G$ is closed if and only if it contains, with any bounded subset $\left\{x_{\alpha}\right\}$, also $\bigvee x_{\alpha}$. Clearly $G$ and 0 are $\ell$-ideals of $G$, they are called improper $\ell$-ideals.

Now in the following theorem we prove that the $\ell$-ideals of any complete $\ell$-group, which are closed in the order topology, is a strongly algebraically closed lattice if it is $q^{\prime}$-compact. 
Theorem 1.3. Let $G$ be a complete $\ell$-group. If the complemented $\ell$-ideals of $G$ is $q^{\prime}$-compact, then it is a strongly algebraically closed lattice.

Proof. Note that if $H$ and $K$ are $\ell$-ideals of the $\ell$-group $G$, then $H \cap K$ and $H+K$ are $\ell$-ideals of $G$. Also we have the complemented $\ell$-ideals of any $\ell$-group $G$ correspond to its direct factors and if $G$ commutative, then its complemented $\ell$-ideals are closed under the polarity defined by disjointness. On the other hand, if $G$ is an $\ell$-group, then an $\ell$-ideal $J \subset G$ is closed if and only if it is complemented. Since any intersection of closed $\ell$-ideals is closed. We will have that the complemented $\ell$-ideals of any complete $\ell$-group form a complete Boolean algebra, hence the complemented $\ell$-ideals of $G$ is $q^{\prime}$-compact and so by applying that ( [10], Theorem 3.5), the complemented $\ell$-ideals of any complete $\ell$-group is a strongly algebraically closed lattice.

\section{Orthomodular lattices and pseudocomplemente semilattices}

Recall that a partially ordered set $(S, \leqslant)$ in which any pair of elements $a$ and $b$ of $S$ has a meet $a \wedge b$ is called (meet) semilattice. A semilattice in which every subset has a meet is actually a complete lattice. A pseudocomplemented semilattice (for short, a $p$-semilattice) is a semilattice $S$ with a least element 0 and a unary operation (the pseudocomplementation) $x \longrightarrow x^{\prime}$ such that, for each $x \in S$,

$$
x \wedge y=0 \Longleftrightarrow y \leqslant x^{\prime}, y \in S,
$$

which is equivalent to saying that for each $x \in S$ there exists a largest element $x^{\prime} \in S$ such that $x \wedge x^{\prime}=0$. Note that any $p$-semilattice has a greatest element $1=0^{\prime}$. For a $p$-semilattice $S$, write

$$
\mathcal{B}(S)=\left\{x^{\prime} \mid x \in S\right\} .
$$

Theorem 2.1. Let $S$ be a p-semilattice. If $S$ is complete lattice and $\mathcal{B}(S)$ is $q^{\prime}$-compact, then $\mathcal{B}(S)$ is a strongly algebraically closed lattice.

Proof. We know that if $S$ is a pseudocomplemented semilattice, then

$$
x \wedge y=x^{\prime \prime} \wedge y^{\prime \prime}=(x \wedge y)^{\prime \prime} \in \mathcal{B}(S),
$$

for $x, y \in S$. Therefore, $\mathcal{B}(S)$ is a semilattice which is clearly dual for the pseudocomplementation inherited from $S$. Using ( [6], Theorem 2.6), $\mathcal{B}(S)$ is a Boolean algebra and by ( [6], Theorem 2.6), $\mathcal{B}(S)$ is a complete Boolean algebra. So, $\mathcal{B}(S)$ is a strongly algebraically closed lattice [10].

An ortholattice is an algebra $L=\left(L, \wedge, \vee,^{\prime}, 0,1\right)$ in which the following holds:

(1) $(L, \wedge, \vee, 0,1)$ is a bounded lattice,

(2) $\left(x^{\prime}\right)^{\prime}=x$,

(3) $(x \vee y)^{\prime}=x^{\prime} \wedge y^{\prime}$,

(4) $x \wedge x^{\prime}=0$,

for all $x, y \in L$.

It is not difficult to see that the equation $(x \wedge y)^{\prime}=x^{\prime} \vee y^{\prime}$ holds in any ortholattice. Boolean algebras are distributive ortholattices. An ortholattice $\left(L, \wedge, \vee,{ }^{\prime}, 0,1\right)$ is called an orthomodular lattice if for all $x, y \in L$ it holds $x \vee y=x \vee\left((x \vee y) \wedge x^{\prime}\right)$.

Let $L$ be a complete orthomodular lattice. A dimensional equivalence relation on $L$ is an equivalence relation $\sim$ which satisfies:

(1) If $a \sim 0$, then $a=0$, 
(2) If $a \leqslant b^{\prime}$ and $c \sim a \vee b$, then $d \leqslant e^{\prime}$ exist such that $d \sim a, e \sim b, c=d \vee e$.

(3) If $\left\{a_{\alpha}\right\}$ and $\left\{b_{\alpha}\right\}$ are families of pairwise orthogonal elements with the same indices $\alpha$, then $a_{\alpha} \sim b_{\alpha}$, for all $\alpha$.

(4) If $a$ and $b$ are perspective (i.e., have common complement in $L$ ), then $a \sim b$.

A lattice orthomodular complete with a dimensional equivalence relation is called a dimension ortholattice. In a dimension ortholattice, an element $e$ is said to be invariant if $a \leqslant e, b \leqslant e^{\prime}$ and $a \sim b$ imply $a=b=0$. Also, we know the invariant elements of a dimension ortholattice is a complete Boolean algebra and also is $q^{\prime}$-compact. So, the collection of the invariant elements of a dimension ortholattice is a strongly algebraically closed lattice. Thus, we obtain the following theorem:

Theorem 2.2. The set of invariant elements of a dimension ortholattice is a strongly algebraically closed lattice if it is $q^{\prime}$-compact.

Acknowledgement. The authors thank the anonymous referees for his/her remarks which helped him to improve the presentation of the paper.

\section{References}

[1] G.Birkhoff, Lattice theory, American Mathematical Society Colloquium Publications, Vol. 25, 3rd ed., American Mathematical Society, New York, 1967.

[2] E.Daniyarova, A.Myasnikov, V.Remeslennikov, Unification theorems in algebraic geometry, Algebra and Discrete Mathamatics, (2008), 80-112.

[3] E.Daniyarova, A.Myasnikov, V.Remeslennikov, Algebraic geometry over algebraic structures, II: Fundations, preprint, arXiv:1002.3562v2[math.AG].

[4] E.Daniyarova, A.Myasnikov, V.Remeslennikov, Algebraic geometry over algebraic structures, III: Equationally Noetherian property and compactness, preprint, arXiv:1002.4243v2 [math.AG].

[5] E.Daniyarova, A.Myasnikov, V.Remeslennikov, Algebraic geometry over algebraic structures, IV: Equatinal domains and co-domains, preprint, 2012.

[6] A.Fernandez Lopez, M.I.Tocon Barroso, Pseudocomplemented semilattices, Boolean algebras, and compatible products 1, Journal of algebra, 242(2001), 60-91.

[7] S.Givant, P.Halmos, Introduction to Boolean algebras, Springer Science + Business Media, New York, 2009.

[8] G.Higman, E.L.Scott, Existentially closed groups, Clarendon Press, 1988.

[9] W.Hodges, Model theory, University Press, Cambridge, 1993.

[10] A.Molkhasi, On strongly algebraically closed lattices, Journal of Siberian Federal University, Mathematics and Physics, 9(2)(2016), 202-208.

[11] R.Sikorski, Boolean algebras, Springer-Verlag, Berlin etc., 1964.

[12] J.Schmid, Algebraically and existentially closed distributive lattices, Zeilschr. Math. Logik und Grundlagen d. Math. Bd., 25(1979), 525-530. 
[13] D.A.Vladimirov, Boolean algebras, Nauka, Moscow, 1969 (in Russian).

[14] L.J.M.Waaijers, On the structure of lattice ordered groups, Waltman, 1968.

\section{Сильно алгебраически замкнутые решетки в $\ell$-группах и полурешетках}

Али Молхаси

Факультет математических наук Университет Табриза, Табриз, 51666-16471

Иран

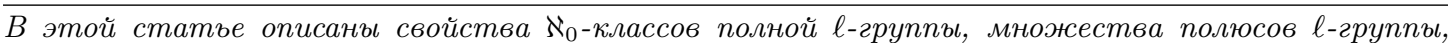
дополненных $\ell$-идеалов полной $\ell$-группы. Изучается множество инвариантных элементов ортостационарной размерности и псевдодополняемые полурешетки с точки зрения теории моделей $u$ получены их отношения к сильно алгебраччески замкнутым решеткам.

Ключевые слова: силъно алгебраччески замкнутые решетки, $\ell$-группы, псевдодополненные полурешетки. 